\title{
Enhanced laser action from smart fabrics made with rollable hyperbolic metamaterials
}

\author{
Hung-I Lin $\mathbb{D}^{1,2,3}$, Chun-Che Wang ${ }^{2}$, Kun-Ching Shen (iD), Mikhail Y. Shalaginov ${ }^{3}$, Pradip Kumar Roy ${ }^{2}$, Krishna Prasad Bera ${ }^{2}$, \\ Monika Kataria ${ }^{2}$, Christy Roshini Paul Inbaraj ${ }^{2}$ and Yang-Fang Chen $\mathbb{D}^{1,2,5 凶}$
}

Rollable photonic devices that can adapt to freeform surfaces with reduced dimensions while maintaining their original functionalities are highly desirable. Among photonic devices, metamaterials with hyperbolic dispersion in momentum space, defined as hyperbolic metamaterial (HMM), possess a large photonic density of states that has been proven to boost light-matter interaction. However, these devices are mainly developed on rigid substrates, restricting their functionalities. Here, we present the attempt to integrate flexible and rollable HMMs consisting of polymer and metal multilayers on paper substrate. Quite interestingly, this design enables to exhibit high photonic density of states and scattering efficiency to enhance stimulated emission and induce pronounced laser action. The flexible and rollable HMM structure remains well its functionalities on freeform surfaces with curvature radius of $1 \mathrm{~mm}$, and can withstand repeated bending without performance degradation. The intensity of laser action is enhanced by 3.5 times as compared to the flat surface. We anticipate that this flexible and rollable HMM structure can serve as a diverse platform for flexible photonic technologies, such as light-emitting devices, wearable optoelectronics, and optical communication.

npj Flexible Electronics (2020)4:20; https://doi.org/10.1038/s41528-020-00085-6

\section{INTRODUCTION}

With the development of photonics, there has been a push towards optoelectronic devices that are flexible, rollable, wearable, user-friendly, and robust to improve human-machine interfaces ${ }^{1,2}$. To be integrated onto human body, these devices must be biocompatible and be able to withstand mechanical deformation and different bending curvatures ${ }^{3,4}$. This promising market of wearable photonics and optoelectronics has inspired a number of demonstrations such as flexible photodetectors ${ }^{5}$, stretchable laser systems ${ }^{6}$, artificial electronic skins ${ }^{3}$, paper-based memory devices $^{7}$, and flexible optoelectronic fibers and textiles ${ }^{8-11}$. The developed flexible photonic devices possess advanced functionalities beyond their rigid counterparts. In order to successfully integrate with wearable photonic systems, the flexible and rollable optoelectronic devices have to contain several important features, such as biocompatibility, high sensitivity, low energy loss, longterm endurance, etc. Undoubtedly, there remains a number of challenges to achieve the above-mentioned functionalities for the realization of practical applications.

Photonic metamaterials offer a number of promising properties suitable for wearable optoelectronic devices. Metamaterials are designed with sub-wavelength dimensions to control or tailor the electromagnetic waves for diverse functionalities ${ }^{12}$. Through the artful design of appropriate geometric patterns, a variety of metamaterials-based structures have been demonstrated, such as epsilon-near zero (ENZ) systems $s^{13,14}$, metaholograms ${ }^{15}$, and metalenses ${ }^{16}$. A special class of metamaterials is a hyperbolic metamaterial (HMM), defined by its hyperbolic iso-frequency surface in momentum space ${ }^{17}$. HMM provides an excellent platform to sustain a large density of high wave-vector modes inside its artificial structure ${ }^{17}$. Subsequently, the photonic density of states (PDOS) is increased as confirmed by the Fermi's golden rule $^{18}$. In general, the compositions of HMMs are alternating metal-dielectric multilayers ${ }^{17}$, ordered arrays of metallic nanowires in a dielectric medium ${ }^{19}$, or the nanoscale core-shell $\mathrm{Au} / \mathrm{SiO}_{2}$ with alternating multishells ${ }^{20}$. HMMs have been used as an extremely sensitive platform for bio-sensors at ultralow diluted concentrations ${ }^{21}$ and reaching a thresholdless Cherenkov radiation to explore the possibility of free-electron light source ${ }^{22}$. As for lightmatter interactions, HMMs have been proven to boost the transition rates for both of the spontaneous and stimulated emission dynamics ${ }^{23-27}$. For spontaneous emission, the nanopatterned structures of HMMs can strongly out-couple the energy with a large enhancement factor ${ }^{28}$. Owing to the increased transition rate of the optical gain media, stimulated emission (e.g., laser action) comes along with a strong output power and a reduced lasing threshold ${ }^{27,29,30}$. However, these demonstrations have primarily been done on rigid substrates. Previously, there exists only one demonstration of flexible HMMs on polyethylene terephthalate (PET) substrate, however the limited curvature radius of $20 \mathrm{~mm}$ reduces its potential application in flexible photonic systems ${ }^{31}$. To fully explore the excellent functionalities of HMM, large degree of flexibility and even rollability are highly desirable, which remains as a challenge issue. Particularly, rollable devices possess several advantages over rigid substrates, such as lighter weight, thinner dimensions, and can be perfectly rolled and stored compactly.

Here, we design and fabricate the flexible and rollable polymerbased HMM on a paper substrate. The flexible and rollable HMM is composed of biocompatible materials: gold $(\mathrm{Au})$ and poly(methyl methacrylate) (PMMA). The constituent materials are arranged in a lamellar multilayer structure to achieve its extremely anisotropic effective permittivity. To realize the usefulness of our design, we demonstrate that the flexible and rollable HMM is able to enhance

\footnotetext{
${ }^{1}$ Graduate Institute of Applied Physics, National Taiwan University, Taipei 106, Taiwan. ${ }^{2}$ Department of Physics, National Taiwan University, Taipei 106, Taiwan. ${ }^{3}$ Department of Materials Science and Engineering, Massachusetts Institute of Technology, 77 Massachusetts Avenue, Cambridge, MA 02139, USA. ${ }^{4}$ Research Center for Applied Sciences, Academia Sinica, Taipei 115, Taiwan. ${ }^{5}$ Advanced Research Center for Green Materials Science and Technology, National Taiwan University, Taipei 106, Taiwan.

凶email: yfchen@phys.ntu.edu.tw
} 
a

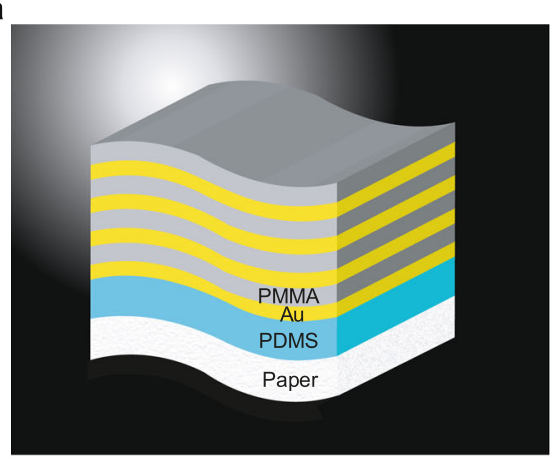

C

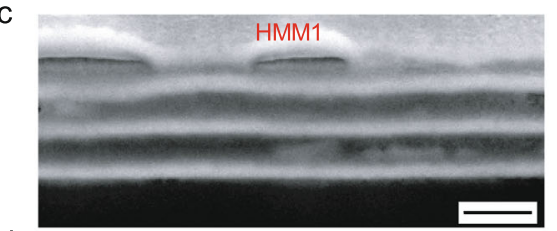

d

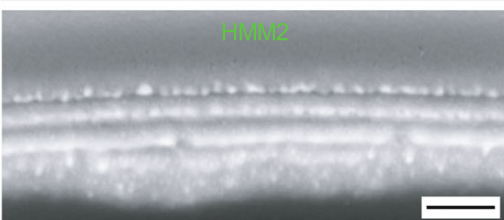

b

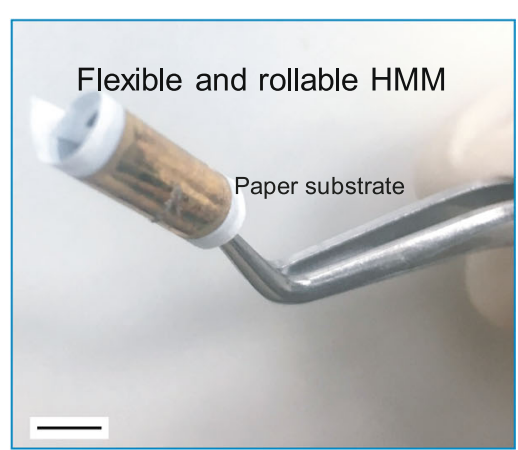

e

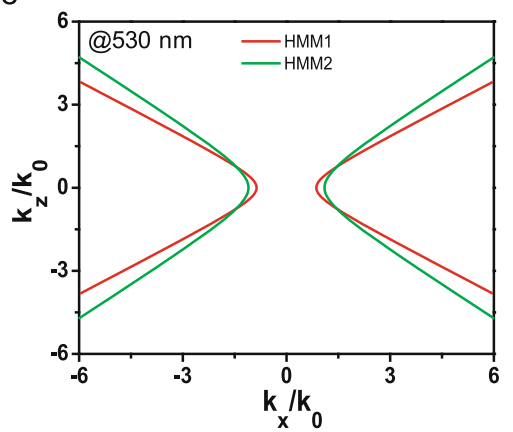

Fig. 1 Proof-of-concept design of the flexible and rollable hyperbolic metamaterials (HMM). a The schematic diagram of the flexible and rollable HMM structure. $\mathbf{b}$ The flexible and rollable HMM device in a rolling configuration. Scale bar represents $1 \mathrm{~cm}$. $\mathbf{c}$, d Cross-sectional fieldemission scanning electron microscopy (FE-SEM) image of the HMM1/HMM2 samples with thicknesses of gold (Au)/poly(methyl methacrylate) (PMMA) for $25 / 40$ and $25 / 30 \mathrm{~nm}$ with four pairs, respectively. Scale bars represent $100 \mathrm{~nm}$. e The iso-frequency curves are plotted at the wavelength of $530 \mathrm{~nm}$ for both of the HMM1 and HMM2 samples.

random laser action, in which light experiences multiple scattering in between disordered media, thereby strongly enhancing its optical gain ${ }^{32-34}$. Advantages of random lasers include cost effective fabrication processes, broad angular emission, cavityfree structure, speckle-free imaging, etc ${ }^{35,36}$. Potential applications for random laser action span from biological probes ${ }^{37}$ and white light illumination ${ }^{29,38}$ to the integration with stretchable and flexible optoelectronic devices ${ }^{6}$. Here, we choose low-dimensional organic-inorganic perovskite nanocrystals (PNCs) as the gain medium. The PNC is composed of methyl-ammonium lead bromide ( $\mathrm{MAPbBr}_{3}$ ) with a bandgap of $\sim 2.3 \mathrm{eV}$, which can achieve a high quantum efficiency up to $\sim 90.5 \%{ }^{39}$ and serve as light outcoupling management in light-emitting diodes ${ }^{40,41}$. Interestingly, the enhanced laser action based on our flexible and rollable HMM maintains superior stable performance even under cyclic bending to curvatures below $1 \mathrm{~mm}$. Finally, when the HMM devices become flexible and rollable, numerous applications can be explored. For example, a highly sensitive bio-sensor can detect the immediate bold glucose on any freeform surfaces of human skin and the biocompatible HMM devices can be implanted into human to enhance the emission of upconversion nanoparticles for in vivo bio-image application. Therefore, the study shown here provides an alternative for the exploration of both scientific research and industrial interest based on the integrations of metamaterials with flexible and rollable technology.

\section{RESULTS}

Proof-of-concept design of the flexible and rollable HMM

To prove the concept of flexible and rollable HMM structure that can be applied to the flexible and rollable applications, Fig. 1a presents the schematic diagram of the flexible and rollable HMM structure on a paper substrate. A thin layer of polydimethylsiloxane (PDMS) was spin-coated on the paper substrate to prevent unwanted roughness due to its textured surface. In order to compare the enhancement factor of the laser action owing to the different hyperbolic dispersion effect of the HMM substrate, we compare two sets of Au/PMMA multilayer with the thickness 25/ $40 \mathrm{~nm}$ (HMM1) as well as $25 / 30 \mathrm{~nm}$ (HMM2) for four pairs. The flexible and rollable HMM device can be easily rolled by a tweezer as shown in Fig. 1b. Figure 1c, d shows the cross-sectional fieldemission scanning electron microscopy (FE-SEM) image of the HMM1 (HMM2) sample, which shows a uniform multilayer structure. To determine the bandwidth of hyperbolic dispersion of the HMM (i.e., $\varepsilon_{\perp} \cdot \varepsilon_{\|}<0$ ), we use the effective medium theory to calculate the optical tensors to match the hyperbolic dispersion ${ }^{17}$ :

$\frac{\omega^{2}}{c^{2}}=\frac{k_{\mathrm{x}}^{2}+k_{\mathrm{y}}^{2}}{\varepsilon_{\|}}+\frac{k_{\mathrm{z}}^{2}}{\varepsilon_{\perp}}$,

where $c$ is the speed of light in vacuum, and the subscripts of $\perp$ and $\|$ are perpendicular and parallel to the anisotropy axis. Figure 1e shows the iso-frequency curves of the flexible and rollable $\mathrm{HMM}$ at the wavelength of $530 \mathrm{~nm}$ to match the central emission wavelength of PNCs. Both of the HMM1 and HMM2 are Type II hyperbolic dispersion ${ }^{42}$. The detailed calculation methods of effective dielectric tensors are shown in Supplementary Note 1 and Supplementary Fig. 1. The enhanced emission dynamics can be explained by the increased PDOS that can out-couple the energy to free space with a higher scattering efficiency.

To realize the effect of thermal expansion mismatch among paper/PDMS/Au, we analyzed the material characterizations as shown in Supplementary Note 2 and Supplementary Fig. 2. The coefficient of thermal expansion (CTE) of PDMS is $3.1 \times 10^{-4}{ }^{\circ} \mathrm{C}^{-143}$, which can cause the deposited Au thin film to have wrinkles on the surface. This unwanted wrinkled Au surface can be avoided by carefully control of sample temperature during deposition process, e.g., we used the thermal evaporation under a vacuum pressure of $\sim 1 \times 10^{-6}$ Torr at room temperature and the deposition rate is 

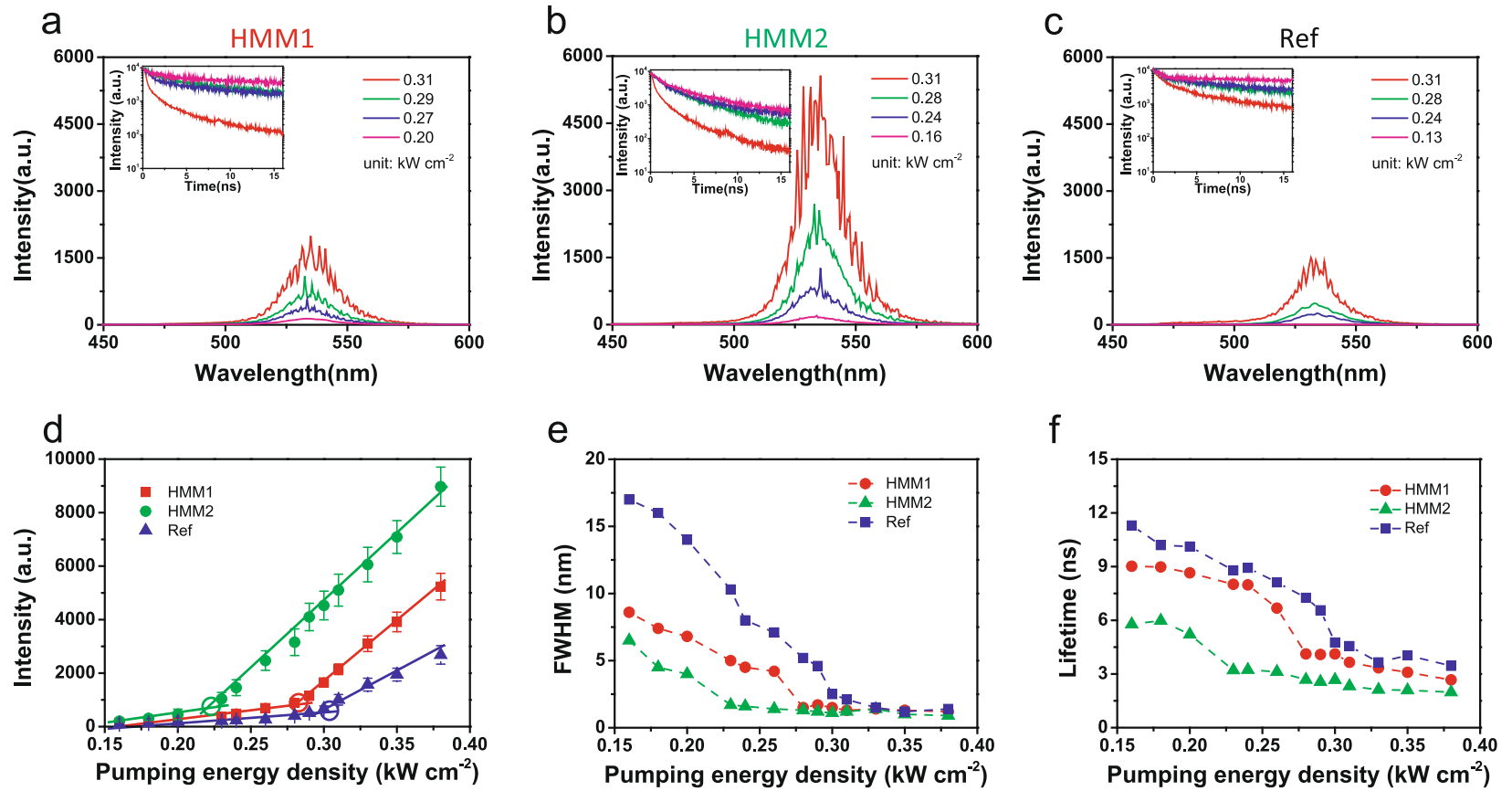

Fig. 2 Emission dynamics of random laser action for the HMM structure. a-c The emission spectra of the PNCs on top of the HMM1, HMM2, and glass substrate, respectively. The emission spectra were measured at four kinds of pumping power density, high power density $\left(0.31 \mathrm{~kW} \mathrm{~cm}^{-2}\right)$, medium power density $\left(0.23-0.29 \mathrm{~kW} \mathrm{~cm}{ }^{-2}\right)$, and low power density $\left(0.13-0.20 \mathrm{~kW} \mathrm{~cm}^{-2}\right)$ to realize the power-dependent emission spectra as a critical realization of the laser action. All the measured emission spectra were excited using a $374 \mathrm{~nm}$ pulsed diode laser. The insets are the corresponding lifetime measurements. $\mathbf{d}$ The maximum lasing peak emission intensity as a function of pumping energy density. The slow rising slope is the amplified spontaneous emission, the drastic slope is the stimulated emission, and the intersection represents the lasing threshold (hollow circle). For the HMM1 (HMM2 and glass substrate), the lasing threshold is 0.27 (0.22 and 0.30 ) $\mathrm{kW}^{\mathrm{cm}}{ }^{-2}$. Error bars are the standard deviation of spectra fluctuation of random laser action from 15 datasets. e The corresponding full width at half maximum (FWHM) and $\mathbf{f}$ the averaged lifetime as a function of pumping energy density.

fixed as slow as $0.5 \AA \mathrm{s}^{-1}$. Then, another layer of PMMA, e.g., for HMM1 is $40 \mathrm{~nm}$ and HMM2 is $30 \mathrm{~nm}$, was spin-coated on the surface of Au not only to serve as the multilayer component of HMM structure but also can be the encapsulation to equalize the residual stress due to CTE mismatch ${ }^{44}$. Furthermore, we spincoated the PNCs onto the HMM devices at a rate of $2000 \mathrm{rpm}$ for $30 \mathrm{~s}$ and then we baked these devices at $50^{\circ} \mathrm{C}$ for $10 \mathrm{~min}$ to volatile toluene. Such low temperature will not affect the thermal expansion of the device.

\section{Emission dynamics}

To explore the properties of the HMM structures, we spin-coated the PNCs on top of the substrates. The high-resolution transmission electron microscopy (HR-TEM) image of the PNC (with a radius of $\sim 10 \mathrm{~nm}$ ) is shown in Supplementary Fig. 3. Figure 2a-c shows the emission spectra of PNCs on the HMM1 (HMM2 and glass substrate). The insets are the corresponding lifetime measurements. All the spectra were measured by a $374 \mathrm{~nm}$ pulsed diode laser at room temperature. The spectra exhibit many randomly distributed sharp peaks, indicating the occurrence of random laser action. The mechanism of random laser action can be realized as follows. The stacking of PNCs with a certain size distribution on top of the substrates provides a platform to trap the light traveling in the PNCs gain media because of multiple scattering, and the high photonic density of states (PDOS) from the HMM substrate is beneficial for the excitation of PNCs. With this feature, the formation of coherent closed loops can occur, the optical gain is increased due to the multiple scattering process in between the PNCs, the weak signal can be enhanced and the spectrum becomes broader ${ }^{45}$. When the optical gain overcomes its loss, lasing peaks with a full width at half maximum (FWHM) less than $1 \mathrm{~nm}$ are observed, which is the signature of random laser action. These spectra reveal that several sharp leasing peaks are randomly emerged with uncertain numbers, positions, and intensities on the top of broad emission bands ${ }^{46}$. The morphologies with randomly distributed PNCs are useful to form the closed-loop paths by trapping the light to further enlarge the optical gain by the happening of multiple scattering process for the emitted photons from PNCs. The stimulated emission of PNCs is subsequently enhanced to achieve the random laser action. Besides, the PNCs on top of the HMM substrate are relatively rough, which is beneficial to extract the trapped photons out of the HMM substrate.

Emission lifetime dynamics are also important for random laser action. Figure $2 \mathrm{~d}$, e show the maximum emission intensity of the lasing peaks and FWHM as a function of pumping energy density. A flat slope with a broad FWHM of $10-15 \mathrm{~nm}$ is typical for spontaneous emission before lasing. When increasing the pumping energy density, the slope climbs sharply and the FWHM of each lasing peak is reduced to less than $1 \mathrm{~nm}$. The intersection of these two slopes (hollow circle) is the lasing threshold for the HMM1 (HMM2 and glass substrate) of 0.27 (0.22 and 0.30) kW $\mathrm{cm}^{-2}$. Note that the lasing threshold for the HMM1 (HMM2) is $10 \%$ $(27 \%)$ reduced and the emission intensity is 1.2 (2.8) times stronger compared with the PNCs on glass substrate. The spatial profiles of random laser systems are quite different than the traditional lasers, which possess with a variety of sharp peaks owing to the existence of many coherent closed loops around the gain media. The calculation method of the temporal coherence length $(L)$ is based on the following formula of $L=c(n \Delta f)^{-1}$, where $c, n$, and $\Delta f$ are the velocity of light at vacuum, the refractive index of the medium, and the frequency bandwidth of the laser action, respectively ${ }^{29}$. Therefore, the temporal coherence length of the laser action is calculated to have a value of $118.8 \mu \mathrm{m}$. Additionally, the differential quantum efficiency ${ }^{47}$ of the HMM1 (HMM2) 

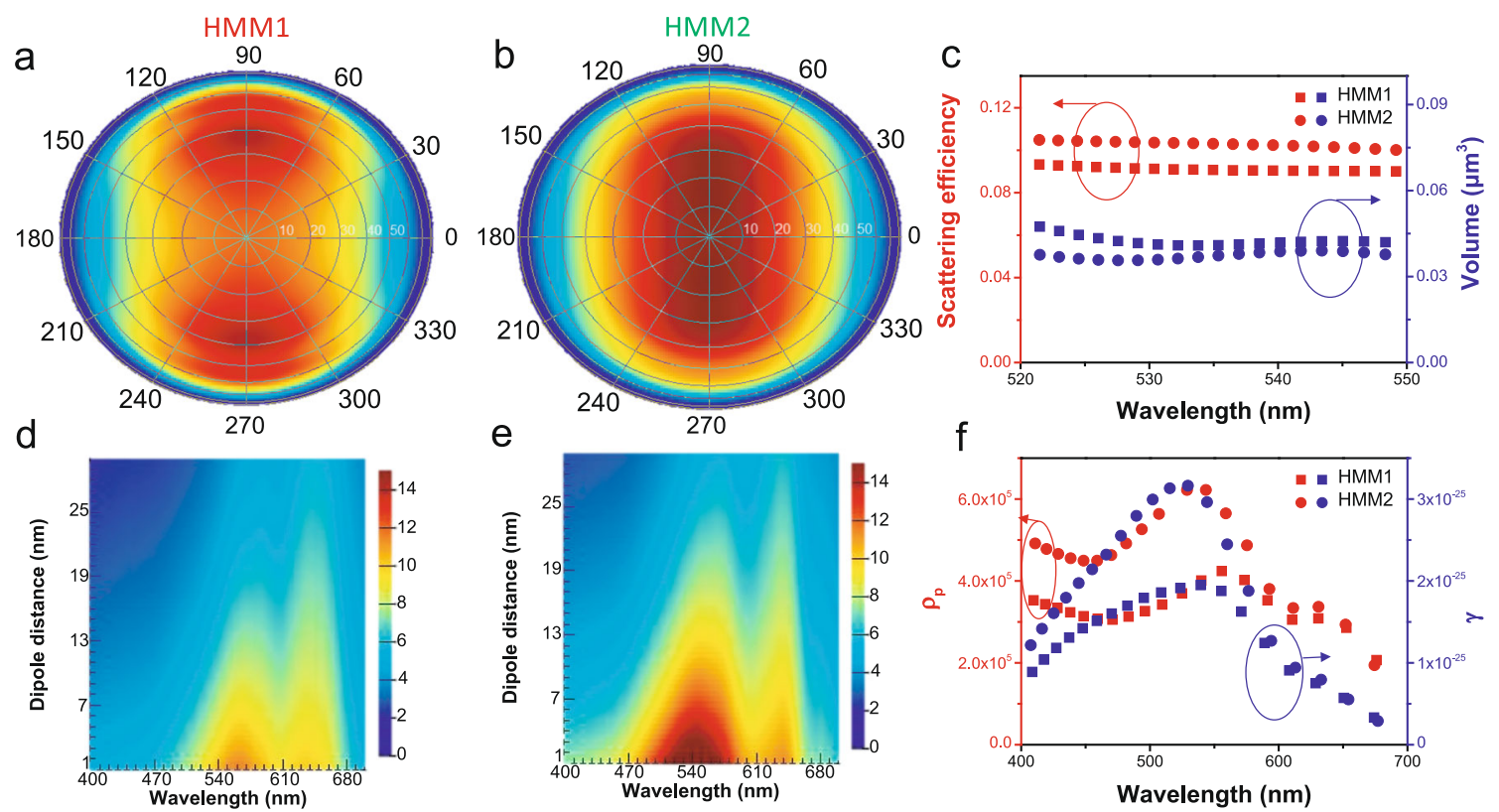

Fig. 3 Theoretical analysis. a, b The far-field angular distributions for the HMM1 and HMM2, respectively. c The scattering efficiency (marked as the red color) and the mode volume (marked as the blue color) of the PNCs on top of the HMM1 and HMM2 substrates. Purcell factors for a perpendicular dipole emitter placed on top of the $\mathbf{d}$ HMM1 and e HMM2 substrates. The dipole-HMM separation distance was varied from 1 to $30 \mathrm{~nm}$. $\mathbf{f}$ The local density of states $\left(\rho_{\mathrm{p}}\right)$ (marked as the red color) and the spontaneous decay rate $(\gamma)$ (marked as the blue color) were calculated by the method of dyadic Green's function. The simulated spectra cover the wavelength range from 400 to $700 \mathrm{~nm}$.

increases 1.4 (2.5) times. When achieving the random laser action, the lifetime is dramatically shortened to $\sim 2 \mathrm{~ns}$, as compared to its spontaneous emission of $\sim 11$ ns as shown in Fig. 2 f.

The effect of metal duty ratio in HMM also plays an important role on the performance of random laser action. Compared with the metal mirror structure, the advantages of HMM are able to integrate with the quantum emitter inside HMM structure to achieve enhanced radiative rate for 20 times and can greatly outcouple light for 100 times to the far-field as compared with typical method less than $1 \%$ of total power ${ }^{48}$. Besides, the different metal duty ratios in HMMs possess the large broadband PDOS with tunable enhancement factors at desired wavelength regions across the visible spectrum. Supplementary Figs 4, 5 show the k-space dissipated power density for different metal duty ratio without and with considering loss effect, respectively. These provide strong evidences to demonstrate that the strong inherent plasmonic modes can exist inside the multilayer structures and then provide the pronounced light-matter interactions, which is very beneficial for the formation of random laser action. Therefore, the above-mentioned benefits provide an excellent platform to extend the applications and functionalities for photovoltaics, ultrafast optics and lasers, and quantum optics.

\section{Theoretical analysis}

It is well known that multiple scattering of a light beam has a high chance of forming closed loop paths ${ }^{34}$. Subsequently, the optical gain is amplified, which is beneficial to achieve random laser action $^{37}$. The discussion of far-field angular distributions as a fundamental realization to further explore the practical applications is necessary, since the devices can be attached to any freeform surfaces on highly flexible and rollable freeform surfaces for the real usages. Figure $3 a, b$ show the far-field angular distribution of the PNCs on the HMM1 and HMM2, respectively. Supplementary Fig. 6 presents the far-field angular electric field intensity $\left(|E|^{2}\right)$ distributions with a similar trend at the normal direction. Since the light is scattered in random directions, we further simulated the tilted angles for $30^{\circ}$ and $60^{\circ}$ to observe the far-field $|E|^{2}$ distributions as shown in Supplementary Fig. 7.
Supplementary Fig. 8 presents the scattering cross-section at different incident angles $(\theta)$ ranging from normal $\left(\theta=0^{\circ}\right)$ to $\theta=$ $33^{\circ}$ in the wavelength region of $520-550 \mathrm{~nm}$ to match the emission wavelength of the PNCs. Briefly, the HMM2 shows a stronger scattering intensity no matter it is an individual PNC, two PNCs or three PNCs as compared with the HMM1 and glass substrate, which is in agreement with our measurements.

Figure $3 c$ presents the scattering efficiency (scattering intensity divided by its scattering cross-sectional area) and the mode volume induced by our designed HMM structures. Within the emission wavelength region of PNCs (520-550 nm), the scattering efficiency for the HMM2 is $16 \%(83 \%)$ higher than the HMM1 (glass substrate). We also calculated the effective mode volume $\left(V_{\text {eff }}\right)$ to investigate the energy confinement. The mode volume is defined as ${ }^{49-51}$ :

$$
V_{\text {eff }}=\frac{\iiint W(r) d^{3}(r)}{\max W(r)}
$$

where $W(r)$ is the energy density of the substrate in free space. Since $V_{\text {eff }}$ is dependent on the energy density, a smaller value of $V_{\text {eff }}$ results from smaller volume confinement. Additionally, the shortened closed loop paths result in the reduction of propagation loss $s^{34}$. The increased energy density for the closed loop paths provides an excellent platform to produce a strong localization of electromagnetic fields around the gain media. The transition probability of the PNCs is therefore enhanced, which enables to trigger the random laser action with a lower lasing threshold. At the wavelength of $530 \mathrm{~nm}$, the mode volume of the glass substrate (HMM1 and HMM2) is 0.123 (0.045 and 0.036) $\mu^{3}$. That is, the mode volume for the HMM2 is $20 \%$ (71\%) smaller than the HMM1 (glass substrate). Supplementary Fig. 9 is the energy distributions of the top-view profiles, showing the HMM2 confines the energy more strongly. Therefore, the reduction of light propagation loss is critical to achieve random laser action.

From the hyperbolic dispersion of iso-frequency curves (Fig. 1d), the realization of the out-coupling configuration is discussed as follows. For both of the HMM samples, the dispersion-relation curves are hyperbolas, where the emission of the PNCs can benefit 
a

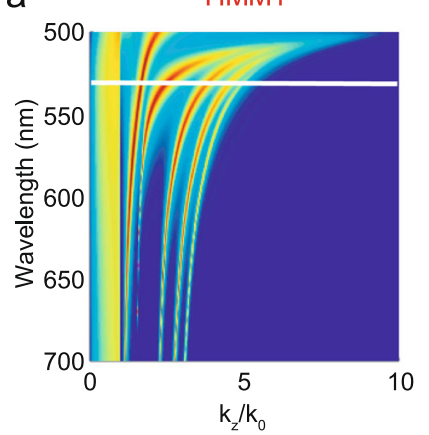

C
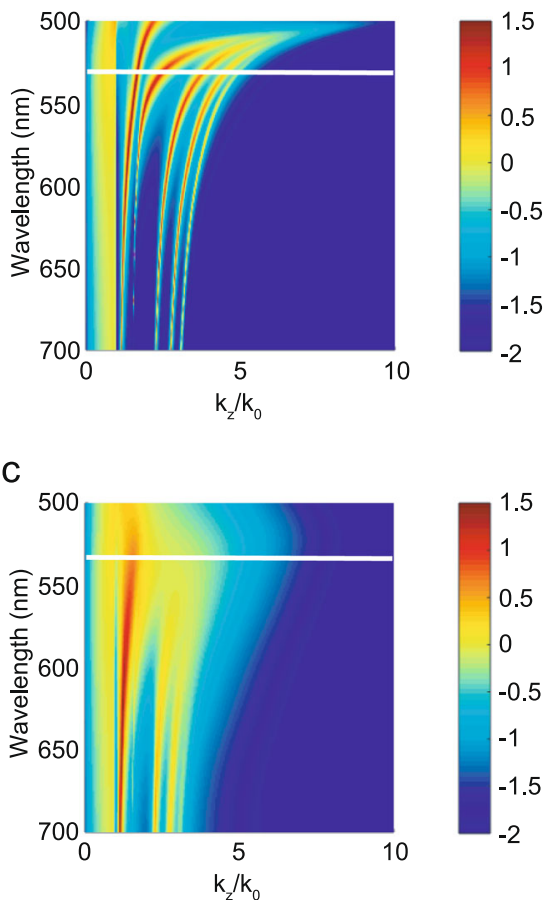

b

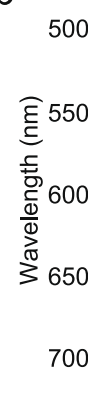

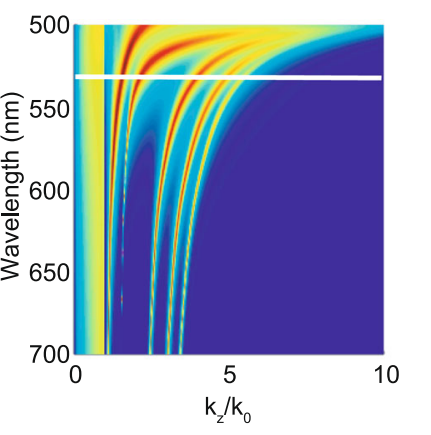

d

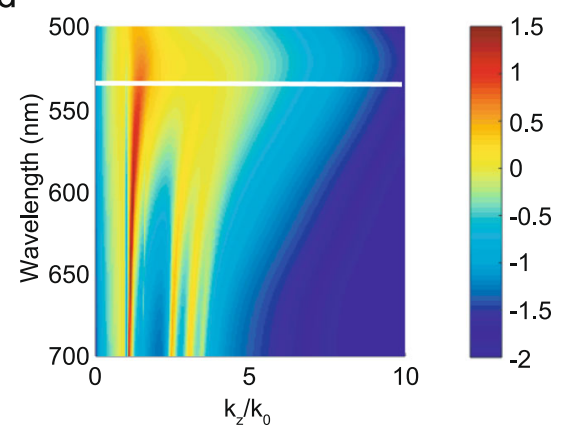

Fig. 4 k-space dissipated power density. The k-space dissipated power density without considering loss effect for the $\mathbf{a}$ HMM1 and $\mathbf{b}$ HMM2. c, d Corresponding dissipated power densities with considering the loss effect. The white line is the emission center of PNCs at the wavelength of $530 \mathrm{~nm}$. For the HMM2 sample, the k-space dissipated power density shows stronger coupling of the emitter to HMM supported plasmonic modes.

from the hyperbolic dispersion of the multilayer structures. Highwavevector modes are known to exist when $\left|k_{\mathrm{HMM}}\right|>\left|k_{\text {air }}\right|$. The proposed multilayer HMM structures are Type II hyperbolic dispersion $\left(\operatorname{Re}\left(\varepsilon_{\perp}<0\right)\right.$ and $\left.\operatorname{Re}\left(\varepsilon_{\mathrm{zz}}\right)>0\right)$, which belongs to side outcoupling. The main advantage of using Type II hyperbolic dispersion is that the edge out-coupling is very beneficial for the formation of closed loops in the in-plane direction to boost the light-matter interaction. Therefore, the coupling effect around the PNCs is enhanced, and then the threshold of laser action is reduced.

To explore the light-matter interaction, we also simulated the Purcell factor $\left(F_{\text {iso }}\right)$ from the perpendicular $\left(F_{\perp}\right)$ and parallel $\left(F_{\|}\right)$ dipole sources ${ }^{52,53}$ :

$F_{\text {iso }}=\frac{1}{3} F_{\perp}+\frac{2}{3} F_{\|}$.

Figure $3 d$, e is the Purcell factors with a perpendicular dipole source above the surface within $30 \mathrm{~nm}$ for the HMM1 (HMM2), while Supplementary Figs. 10a, 10b is the corresponding parallel dipole source. Since the thickness of the PNCs is only $\sim 20 \mathrm{~nm}$, the influence of Purcell effect from the surface is very pronounced. The HMM2 shows 60\% higher Purcell factor than the HMM1 at the wavelength of $530 \mathrm{~nm}$, which can be attributed to the excitation of a higher PDOS. To confirm the above-mentioned concept, we further simulated the local density of states (LDOS) and spontaneous decay rate as shown in Fig. $3 f$, the method of dyadic Green's function $^{54}$. At the wavelength of $530 \mathrm{~nm}$, the LDOS and spontaneous decay rate for the HMM2 are 57 and $62 \%$ higher than the HMM1, respectively, which are very similar to the enhancement from the Purcell effect and the differential quantum efficiency obtained from emission spectra. Since the Purcell effect, LDOS, and spontaneous decay rate are achieved the strongest factors at the emission wavelength of PNCs, the composition of HMM2 structure can be considered as the suitable candidate to enhance the laser action. Furthermore, to prove the influence of
Purcell effect, the $\mathbf{k}$-space dissipated power density without considering loss effect is calculated as shown in Fig. $4 a$, b for the $\mathrm{HMM} 1$ and $\mathrm{HMM} 2$, respectively. In addition, Fig. $4 \mathrm{c}$, $d$ are the corresponding dissipated power densities with considering the loss effect. At the wavelength of $530 \mathrm{~nm}$, especially for the HMM2, these k-space dissipated power densities show that the strong inherent plasmonic modes can exist inside the multilayer structures. These plasmonic modes provide the pronounced Purcell enhancement effect, which is beneficial for the formation of random laser action.

\section{Functionalities on freeform surfaces}

To explore the curvature realization of the flexible and rollable HMM, we integrated this HMM device on freeform surfaces for flexible and wearable characterizations. Figure 5 shows the emission spectra of random lasing action for the HMM2 device on the concave glass substrates under the curvature radius of $20 \mathrm{~mm}$ (Fig. 5a) and $50 \mathrm{~mm}$ (Fig. 5b), and on the convex glass substrates under the curvature radius of $20 \mathrm{~mm}$ (Fig. $5 \mathrm{c}$ ) and $50 \mathrm{~mm}$ (Fig. $5 \mathrm{~d}$ ). To demonstrate that our HMM is wearable, we also integrate the flexible and rollable HMM on a glove as shown in Supplementary Fig. 11, in which the pronounced laser action can still be seen. The emission spectra on a convex glass bottle with the radius curvature of $20 \mathrm{~mm}$ (Supplementary Fig. 12) shows the similar enhancements. All the measurements were performed under the same pumping energy density of $0.24,0.20$, and $0.13 \mathrm{~kW} \mathrm{~cm}^{-2}$ to observe the trends of emission intensities. For the difference in intensity between Fig. 5c and Supplementary Fig. 12, it is due to the measurements were at different positions. As shown in Fig. 5c, the laser spot is located near the scratch of the HMM structure on the convex glass substrates under the curvature radius of $20 \mathrm{~mm}$, which is similar to the emission intensities at the same position on a convex glass bottle under the same curvature radius (Supplementary Fig. 12a). However, the emission intensities could drop for $41.7 \%$ when the laser spot is located on the off-axis 

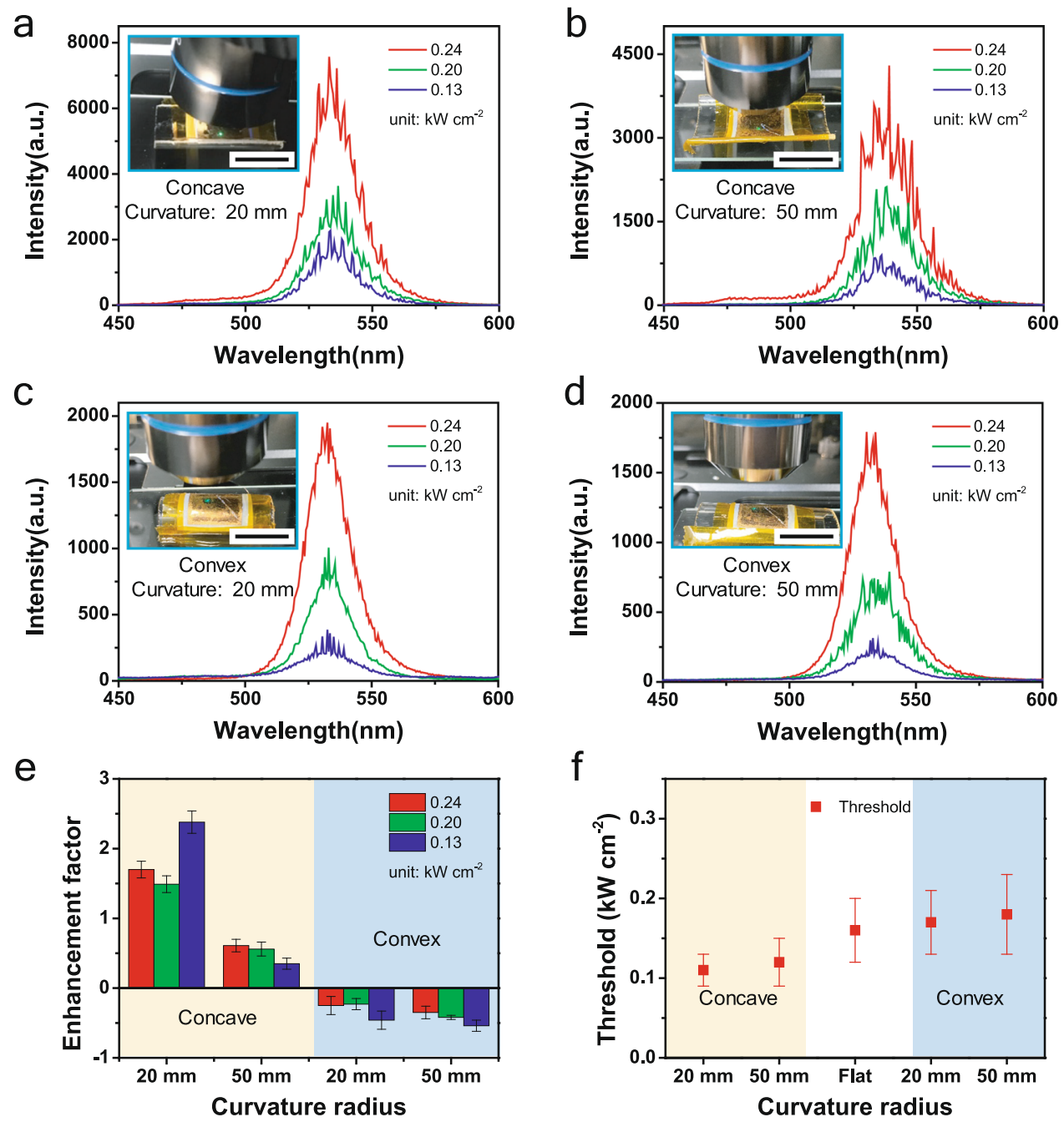

Fig. 5 Curvature realization of the flexible and rollable HMM device. The emission spectra of random lasing action for the HMM2 device on the concave glass substrates under the curvature radius of a $20 \mathrm{~mm}$ and b $50 \mathrm{~mm}$ with the corresponding insets. c, $\mathbf{d}$ The emission spectra on the convex glass substrates under the curvature radius of 20 and $50 \mathrm{~mm}$, respectively. The insets are the corresponding photo images. e The enhancement factors of concave and convex substrates as compared with the planar substrate. $f$ The threshold variations with the curvature radius for the flexible and rollable HMM structure on concave and convex substrates. Error bars are the standard deviation of spectra fluctuation of random laser action from 15 datasets. All the measurements were performed under the same pumping energy density of 0.24 , 0.20 , and $0.13 \mathrm{~kW} \mathrm{~cm}^{-2}$. All the scale bars are $1 \mathrm{~cm}$.

surface (Supplementary Fig. 12b). Figure 5e shows the enhancement factors of concave and convex substrates as compared with the planar substrate. The threshold variations with the curvature radius on the concave, flat, and convex substrates is shown in Fig. $5 f$. As compared to the flat surface, the threshold is $\sim 28.2 \%$ lower than on the concave substrate and is $\sim 9.5 \%$ higher than on the convex substrate. This is due to the capability of the concave structure to trap the light through multiple reflections inside the substrate, which provides an easier way to form feedback loops to achieve random laser action ${ }^{55}$. Especially for the curvature of $20 \mathrm{~mm}$ indicates more light can induce the photon trapping inside the concave substrate, which in turn greatly reduces the loss of substrate-induced-absorption during the multiple reflections, and then leads to the amplification of stimulated emission from $\mathrm{PNCs}^{55}$. On the contrary, for the decayed emission intensities on the convex substrates, this phenomenon is due to the less scattering process in the PNCs and the effect of the PDOS from the HMM becomes less on the convex substrates to achieve laser action. Our measured results show that the laser action of PNCs on the flexible and rollable HMM remains unaffected despite mechanical deformation. The demonstration of flexible and wearable characteristics is important to advance the applications of optoelectronic devices ${ }^{56-59}$.

We further demonstrate the functionality of our developed HMM structures as a feasible rollable photonics usage. Figure $6 a$ and Supplementary Fig. 13a, b show the lasing performance of several rolling cycles under the pumping energy density of 0.24 , 0.20 , and $0.13 \mathrm{~kW} \mathrm{~cm}^{-2}$, respectively, indicating the stability of the devices. Each cycle contains the rolling process (Fig. 6b) and flattening after rolling (Fig. $6 \mathrm{c}$ ). The curvature radius of the HMM under rolling is $1 \mathrm{~mm}$. The repeated rolling-flattening processes were performed with care to prevent the fall off the perovskite nanocrystals by accident. We further performed the measurements of random lasing action up to 50 times under the pumping energy density of $0.24,0.20$ and $0.13 \mathrm{~kW} \mathrm{~cm}^{-2}$ as shown in Fig. $6 \mathrm{~d}$. The emission shows an excellent stability without obvious photodegradation. This indicates that the flexible and rollable HMM structure shows the higher emission intensities for the flattening state as compared to the rolling state, in which the light will be dissipated on the rolling side of the HMM structure. The fluctuation of spectra is the typical feature of random laser action. On the other hand, the results from Fig. 5 are the measurements 


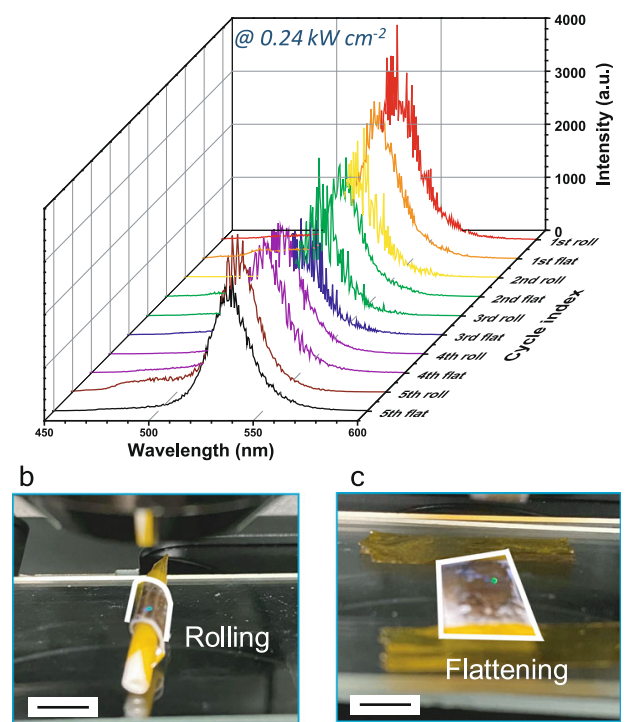

d

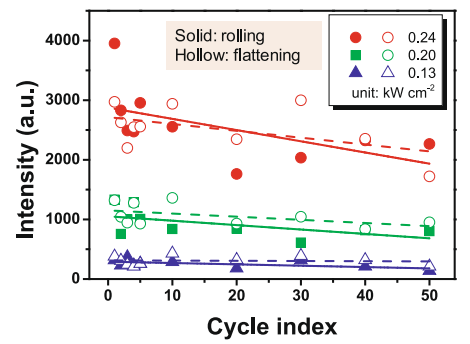

e

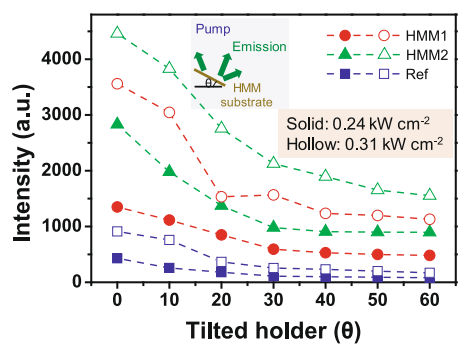

f

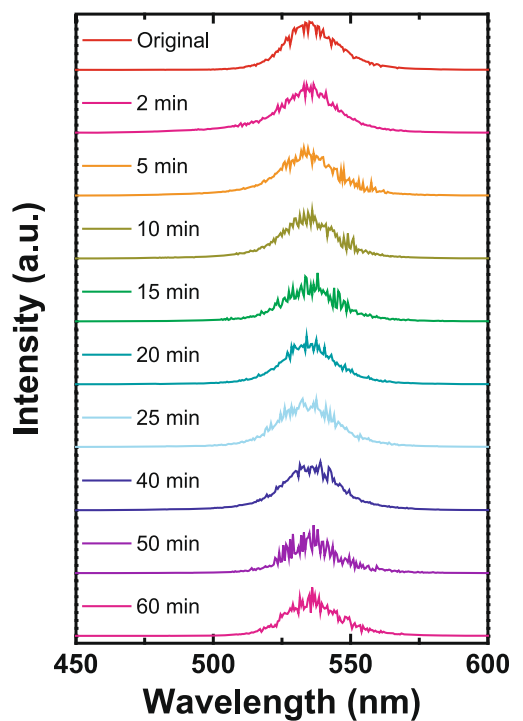

Fig. 6 Functionalities of the flexible and rollable HMM device. a The emission spectra of random lasing action for the HMM2 device with increasing cycle index at the pumping energy density of $0.24 \mathrm{~kW} \mathrm{~cm}{ }^{-2}$. The photo image of the rollable HMM assisted by a wood stick with a diameter of $1 \mathrm{~mm}$ (yellow color) $\mathbf{b}$ under rolling and $\mathbf{c}$ flattening. Scale bars represent $3 \mathrm{~mm}$. $\mathbf{d}$ The emission intensities of random lasing action of repeated rolling-flattening processes up to 50 times under the pumping energy density of $0.24,0.20$, and $0.13 \mathrm{~kW} \mathrm{~cm}^{-2}$. The solid straight line is the fitted results for the rolling state and the dash straight line is for the flattening state. e The emission intensities measured from normal to tilted sample holder of $60^{\circ}$ under the pumping energy density of 0.24 and $0.31 \mathrm{~kW} \mathrm{~cm}^{-2}$. Inset is the schematic diagram of the measuring process for the tilted sample holder. $\mathbf{f}$ The lasing spectra with laser expose of PNCs on HMM2 substrate for continuously 60 min at the pumping energy density of $0.24 \mathrm{~kW} \mathrm{~cm}^{-2}$.

of steady flexible and rollable HMM structure on the concave or convex substrates without suffering any repeated deformation, resulting in the less possible degradation of the HMM structure inside the multilayer components. Hence the emission intensities in Fig. 5 show the relative stable emission intensities. Supplementary Fig. 14 show the corresponding lasing spectra with further increasing cycle index up to 50 times. Since the PNCs are decorated on the rollable HMM, the rolling structure may provide a feasible manner to trap light in the rolled device for coherent laser action to occur more easily, and resulting in more pronounced sharp peaks. In addition, the mode volume of the rollable HMM is reduced with decreasing curvature radius, which is beneficial to enhance scattering and reduce propagation loss. Ultimately, this cyclic testing shows that our devices may be integrated on frequently deformed substrates without device performance degradation, which is very beneficial for the practical fabrics usage owing to its excellent tolerance property in mechanical force. Figure $6 \mathrm{e}$ shows broad angular emissions intensities from $10^{\circ}$ to $60^{\circ}$ and the trend is very similar to the normalized intensity of far-field angular emission at the center position of far-field monitor (Supplementary Fig. 15) and Supplementary Fig. 16 shows the corresponding spectra, which is another characteristic to firm the occurrence of random laser action. Certainly, the spectra of far-field angle emission are simply changing the tilted angle of sample holder, and the detected beam is collected by the same objective lens (Fig. 6e). These spectra were collected by the Horiba Jobin Yvon TRIAX 320 spectrometer with 50x (Olympus, Japan), in which the emissions are reflected by the mirrors many times before it reaches to the optical fiber and then to the detector systems (Supplementary Fig. 17). To further demonstrate the broad angular emissions, we perform the measurements at different emission directions as shown in Supplementary Fig. 18, where the emissions are collected by an optical fiber near the HMM sample and then the spectra are analyzed by the OCEAN OPTICS HR2000 + CG. Note that the detector used to measure the different emission directions is different than the Horiba Jobin Yvon TRIAX
320 spectrometer, as well as the numbers of passing through or reflected by the mirrors are different. The laser action can be observed up to $50^{\circ}$ under the pumping energy density of $0.24 \mathrm{~kW} \mathrm{~cm}^{-2}$.

We performed the laser exposure to observe the morphology of PNCs as shown in Supplementary Note 3 and Supplementary Fig. 19. We can observe the stability of PNCs without any decomposition or degradation on the HMM2 substrate even under continuously laser expose at pumping energy density of $0.24 \mathrm{~kW} \mathrm{~cm}^{-2}$ for $60 \mathrm{~min}$ of the laser irradiation. The temperature at laser spot only increases $0.6^{\circ} \mathrm{C}$ (in Supplementary Fig. 20). This slightly increment of temperature may be attributed to the fact that both of the HMM1 and HMM2 samples are composed of $\mathrm{Au}$ thin layer up to $100 \mathrm{~nm}$ in total thickness, which could dissipate the heat accumulation in the interface between PNCs and HMM samples ${ }^{60}$. In general, the PNCs present unstable performances above $85^{\circ} \mathrm{C}$ even in the inert atmosphere ${ }^{61}$. So the laser source induced degradation can be neglected in our measurement of random laser action. Furthermore, we performed the measurement with laser exposure of PNCs on HMM2 substrate for continuously $60 \mathrm{~min}$ at the pumping energy density of $0.24 \mathrm{~kW}$ $\mathrm{cm}^{-2}$ as shown in Fig. 6f. No spectral shifts are observed, demonstrating that the PNCs remain intact without any decomposed resulting in the higher binding energy during the laser irradiation ${ }^{60,62}$.

The HMM device, i.e., flexible and rollable polymer-based HMM, is composed of Au and PMMA, which are biocompatible materials, including the paper substrate. $\mathrm{MAPbBr}_{3}$ was spin-coated on the flexible and rollable HMM device to perform the measurement of random laser action to prove the preliminary function to enhance the light-matter interaction. Note that the flexible and rollable HMM on a paper substrate can serve as an alternative towards smart fabrics with the following anticipation. First, the flexible and rollable HMM is composed of the high elasticity and super hydrophobicity polymers that makes it to integrate with the fabrics materials as a firm, impermeable and wear-resistant photonic skin (p-skin) and electronic skin (e-skin) component. 
Eco-friendly and disposable characterizations, low-cost fabrication, lightweight, and the control of surface wettability and surface free energy to eliminate the external flow effect from the atmosphere as a packaging protection, the chosen paper as a substrate is foreseeable for the development of rollable photonics. Since the ultra-sensitivity of the nano-patterned HMM structure, this device on a paper substrate can be even used for in vivo implantable biosensors and detecting explosive molecules on freeform surface. Finally, the demonstration of this work provides an excellent platform to widely explore the research highlights for rollable photonics and smart fabrics.

\section{DISCUSSION}

We have successfully demonstrated HMM structures composed of Au/PMMA on paper substrate with flexible and rollable characteristics. This flexible and rollable HMM structures show hyperbolic dispersion in the momentum space to enlarge the PDOS, which enables to enhance the optical gain and boost the stimulated emission (e.g., random laser action) from PNCs. By tuning the thickness of the HMM structure, we have achieved a large enhancement of the density of states and the scattering efficiency. Simulation results based on the scattering efficiency and the dipole-like dynamics confirm an efficient out-coupling. Remarkably, owing to its flexibility and rollability, the HMM structure still works well on freeform surfaces with curvature radius of $1 \mathrm{~mm}$ during cyclic testing. Our demonstration of flexible and rollable HMM structures therefore provides a great promise for applications in multifunctional and wearable optoelectronic devices.

\section{METHODS}

Fabrication of the flexible and rollable HMM

We used a paper as our flexible and rollable HMM substrate. Paper with size of $\sim 2 \mathrm{~cm} \times 2 \mathrm{~cm}$ is attached to the glass substrate for the following spin-coating process. To avoid the unwanted roughness from the textured surface of the paper substrate, a thin layer of PDMS was spin-coated on the paper/glass substrate at a rate of $2000 \mathrm{rpm}$, and then the sample was cured for $40 \mathrm{~min}$ at $100^{\circ} \mathrm{C}$ on a hotplate. After that, to prepare the multilayer structures, we deposited Au thin film on the top of PDMS layer using the thermal evaporation under a vacuum pressure of $\sim 1 \times 10^{-6}$ Torr at the deposition rate of $0.5 \AA \mathrm{s}^{-1}$. We dissolved PMMA into acetone at a mass fraction of $25 \mathrm{wt} \%$ and then stirred this solution for $2 \mathrm{~h}$ at room temperature to obtain a homogeneous mixture. To prepare the HMM1 and HMM2 with different thickness of PMMA, we spin-coated the solution at a rate of $3000(5000) \mathrm{rpm}$ to get the thickness of $40(30) \mathrm{nm}$, and then cured the sample for $20 \mathrm{~min}$ at $100^{\circ} \mathrm{C}$ on a hotplate. We continuously repeated this process for three times to obtain four pairs of HMM1 and HMM2 devices.

\section{Materials characterization of the flexible and rollable HMM}

The characterization of the multilayer structures of the HMM1 and HMM2 devices was performed by the focused ion beam (FIB) system (Helios Nanolab 660 DualBeam) at $30 \mathrm{kV}$ and $50 \mathrm{pA}$ of Gallium ion source. Then, we used the FE-SEM system to record the images at a tilted holder angle of $52^{\circ}$.

Measurement of emission spectra and the corresponding lifetime The emission spectra of random laser action were measured by a pulsed diode laser (Picoquant, PDL 800-B) with a central wavelength of $374 \mathrm{~nm}$, pulsed duration of $70 \mathrm{ps}$ and repetition rate of $2.5 \mathrm{MHz}$. The emission spectra and lifetime dynamics were recorded by a Horiba Jobin Yvon TRIAX 320 spectrometer with $50 \times$ (Olympus, Japan) objective. The resolution of the spectrometer is $0.1 \mathrm{~nm}$. Since the laser action is related to the dynamics of charge carrier mechanism, we used the time-correlated single photon counting (TCSPC) for data acquisition to perform the lifetime measurements, which is a commonly used method. TCSPC is to measure the time difference between the excitation signal from the sample and the emitted photons arrival to the detector. Finally, the lifetime was estimated using the fitting software (FluoFit, PicoQuant). This measurement is repeated under different pumping density to realize the lifetime of random laser action. We also used OCEAN OPTICS HR2000+CG with a $0.47 \mathrm{~nm}$ in spectral resolution to measure the different emission directions from the HMM sample.

\section{Preparation of the PNCs}

We repeated the method from the previous report to synthesize the $\mathrm{CH}_{3} \mathrm{NH}_{3} \mathrm{PbBr}_{3} \mathrm{PNCs}^{63}$. First, $96 \mu \mathrm{L}$ of oleic acid was mixed with $2 \mathrm{~mL}$ of octadecene, and then this solution was stirred and heated at $80^{\circ} \mathrm{C}$ on a hotplate. Next, $0.06 \mathrm{mmol}$ of octylammonium bromide (Shanghai MaterWin New Materials) was added into this solution and then another $200 \mu \mathrm{L}$ of $\mathrm{N}, 2$-dimethylformamide. $0.04 \mathrm{mmol}$ of methylammonium bromide (Shanghai MaterWin New Materials) was then added into the solution followed by the mixture of $0.1 \mathrm{mmol}$ of lead(II) bromide. This as synthesized PNCs possesses an extremely strong photoluminescence, which is necessary for laser action on the HMM device. To integrate the PNCs onto the device, we dissolved the PNCs into toluene with a concentration of $10 \mathrm{mg} \mathrm{mL}^{-1}$ at room temperature followed by stirring it for $1 \mathrm{~h}$ to get a homogenous mixture. Then, we spin-coated this solution on the HMM devices at a rate of $2000 \mathrm{rpm}$ for $30 \mathrm{~s}$. Finally, we baked these devices at $50^{\circ} \mathrm{C}$ for $10 \mathrm{~min}$ to volatile toluene.

\section{Instrumentation detail of HR-TEM}

The HR-TEM image of the as-synthesized PNCs was carried out using TECNAI G2 FEG-TEM operating at $300 \mathrm{kV}$.

\section{Numerical simulation}

The simulation results were performed by using a commercial electromagnetic software (Lumerical) of finite-difference time-domain (FDTD) Solutions. The refractive index of PMMA was set to be 1.49 and Au was from Johnson and Christy ${ }^{64}$. Since the PNCs used is composed of $\mathrm{CH}_{3} \mathrm{NH}_{3} \mathrm{PbBr}_{3}$, so we used the complex refractive indices $(n+i k)$ of refractive index $(n)$ and extinction coefficient $(k)$ from Alias et al. as shown in Supplementary Fig. $21^{65}$. At the emission center of PNCs at the wavelength of $530 \mathrm{~nm}$, the $n$ and $k$ are 2.1 and 0.2 , respectively. To avoid the unwanted numerical results owing to the boundary conditions, we used the perfectly matched layers to cover the entire computational regions. The simulation settings of far-field angular distribution, scattering and Purcell factors (from Supplementary Notes 4-7 and from Supplementary Figs. 22-26).

\section{DATA AVAILABILITY}

The data that support the findings of this study are available from the corresponding author upon reasonable request.

Received: 26 November 2019; Accepted: 10 July 2020; Published online: 02 September 2020

\section{REFERENCES}

1. Rogers, J. A., Someya, T. \& Huang, Y. Materials and mechanics for stretchable electronics. Science 327, 1603-1607 (2010).

2. Liu, F., Song, S., Xue, D. \& Zhang, H. Folded structured graphene paper for high performance electrode materials. Adv. Mater. 24, 1089-1094 (2012).

3. Hammock, M. L., Chortos, A., Tee, B. C. K., Tok, J. B. H. \& Bao, Z. 25th anniversary article: the evolution of electronic skin (e-skin): a brief history, design considerations, and recent progress. Adv. Mater. 25, 5997-6038 (2013).

4. Cai, S.-Y. et al. Ultrahigh sensitive and flexible magnetoelectronics with magnetic nanocomposites: toward an additional perception of artificial intelligence. ACS Appl. Mater. Interfaces 10, 17393-17400 (2018).

5. Lin, S.-Y. et al. Transient and flexible photodetectors. ACS Appl. Nano Mater. 1, 5092-5100 (2018).

6. Liao, Y.-M. et al. Highly stretchable label-like random laser on universal substrates. Adv. Mater. Technol. 1, 1600068 (2016).

7. Lien, D.-H. et al. All-printed paper memory. ACS Nano 8, 7613-7619 (2014).

8. Zeng, W. et al. Fiber-based wearable electronics: a review of materials, fabrication, devices, and applications. Adv. Mater. 26, 5310-5336 (2014).

9. Yan, W. et al. Advanced multimaterial electronic and optoelectronic fibers and textiles. Adv. Mater. 31, 1802348 (2019). 
10. Yan, W. et al. Thermally drawn advanced functional fibers: New frontier of flexible electronics. Mater. Today 35, 168-194 (2020).

11. Loke, G., Yan, W., Khudiyev, T., Noel, G. \& Fink, Y. Recent progress and perspectives of thermally drawn multimaterial fiber electronics. Adv. Mater. 32, 1904911 (2020).

12. Fang, N., Lee, H., Sun, C. \& Zhang, X. Sub-diffraction-limited optical imaging with a silver superlens. Science 308, 534-537 (2005).

13. Cao, T., Zou, Y., Adawi, A. M. \& Cryan, M. J. Directive emission of red conjugated polymer embedded within zero index metamaterials. Opt. Express 22, 22699-22706 (2014).

14. Alù, A., Silveirinha, M. G., Salandrino, A. \& Engheta, N. Epsilon-near-zero metamaterials and electromagnetic sources: tailoring the radiation phase pattern. Phys. Rev. B 75, 155410 (2007).

15. Huang, Y.-W. et al. Aluminum plasmonic multicolor meta-hologram. Nano Lett 15, 3122-3127 (2015)

16. Khorasaninejad, $M$. et al. Metalenses at visible wavelengths: diffraction-limited focusing and subwavelength resolution imaging. Science 352, 1190-1194 (2016).

17. Poddubny, A., lorsh, I., Belov, P. \& Kivshar, Y. Hyperbolic metamaterials. Nat. Photonics 7, 948-957 (2013).

18. Jacob, Z. et al. Engineering photonic density of states using metamaterials. Appl. Phys. B 100, 215-218 (2010).

19. Wu, C., Salandrino, A., Ni, X. \& Zhang, X. Electrodynamical light trapping using whispering-gallery resonances in hyperbolic cavities. Phys. Rev. X 4, 021015 (2014).

20. Wang, P. et al. Metaparticles: dressing nano-objects with a hyperbolic coating Laser Photonics Rev. 12, 1800179 (2018).

21. Sreekanth, K. V. et al. Extreme sensitivity biosensing platform based on hyperbolic metamaterials. Nat. Mater. 15, 621-627 (2016).

22. Liu, F. et al. Integrated cherenkov radiation emitter eliminating the electron velocity threshold. Nat. Photonics 11, 289-292 (2017).

23. Chandrasekar, R. et al. Lasing action with gold nanorod hyperbolic metamaterials. ACS Photonics 4, 674-680 (2017).

24. Shen, K.-C., Hsieh, C., Cheng, Y.-J. \& Tsai, D. P. Giant enhancement of emission efficiency and light directivity by using hyperbolic metacavity on deep-ultraviolet algan emitter. Nano Energy 45, 353-358 (2018).

25. Yang, X., Yao, J., Rho, J., Yin, X. \& Zhang, X. Experimental realization of threedimensional indefinite cavities at the nanoscale with anomalous scaling laws. Nat. Photonics 6, 450-454 (2012).

26. Cortes, C. L., Newman, W., Molesky, S. \& Jacob, Z. Quantum nanophotonics using hyperbolic metamaterials. J. Opt. 14, 063001 (2012).

27. Lin, H.-I. et al. Integration of nanoscale light emitters and hyperbolic metamaterials: an efficient platform for the enhancement of random laser action. ACS Photonics 5, 718-727 (2018)

28. Lu, D., Kan, J. J., Fullerton, E. E. \& Liu, Z. Enhancing spontaneous emission rates of molecules using nanopatterned multilayer hyperbolic metamaterials. Nat. Nanotechnol. 9, 48-53 (2014).

29. Haider, G. et al. A highly-efficient single segment white random laser. ACS Nano 12, 11847-11859 (2018).

30. Shen, K.-C. et al. Deep-ultraviolet hyperbolic metacavity laser. Adv. Mater. 30, 1706918 (2018)

31. Lin, H.-I. et al. Transient and flexible hyperbolic metamaterials on freeform surfaces. Sci. Rep. 8, 9469 (2018).

32. Letokhov, V. S. Generation of light by a scattering medium with negative resonance absorption. Sov. J. Exp. Theor. Phys. 26, 835-840 (1968).

33. Andreasen, J. et al. Modes of random lasers. Adv. Opt. Photonics 3, 88-127 (2010).

34. Liu, J. et al. Random nanolasing in the anderson localized regime. Nat. Nanotechnol. 9, 285-289 (2014).

35. Wiersma, D. S. \& Lagendijk, A. Light diffusion with gain and random lasers. Phys. Rev. E 54, 4256-4265 (1996).

36. Cheng-Yen, T. et al. Magnetically controllable random lasers. Adv. Mater. Technol. 2, 1700170 (2017).

37. Wiersma, D. S. The physics and applications of random lasers. Nat. Phys. 4, 359-367 (2008).

38. Chang, S.-W. et al. A white random laser. Sci. Rep. 8, 2720 (2018)

39. Lu, C.-H., Hu, J., Shih, W. Y. \& Shih, W.-H. Control of morphology, photoluminescence, and stability of colloidal methylammonium lead bromide nanocrystals by oleylamine capping molecules. J. Colloid Interface Sci. 484 17-23 (2016).

40. Zhang, Q. et al. Efficient metal halide perovskite light-emitting diodes with significantly improved light extraction on nanophotonic substrates. Nat. Commun. 10, 727 (2019)

41. Zhang, Q. et al. Three-dimensional perovskite nanophotonic wire array-based light-emitting diodes with significantly improved efficiency and stability. ACS Nano 14, 1577-1585 (2020).
42. Ferrari, L., Smalley, J. S. T., Fainman, Y. \& Liu, Z. Hyperbolic metamaterials for dispersion-assisted directional light emission. Nanoscale 9, 9034-9048 (2017).

43. Buma, T., Spisar, M. \& O'Donnell, M. High-frequency ultrasound array element using thermoelastic expansion in an elastomeric film. Appl. Phys. Lett. 79, 548-550 (2001).

44. Walia, S. et al. Flexible metasurfaces and metamaterials: A review of materials and fabrication processes at micro- and nano-scales. Appl. Phys. Rev. 2, 011303 (2015).

45. Cao, H. et al. Random laser action in semiconductor powder. Phys. Rev. Lett. 82, 2278-2281 (1999).

46. Sun, T.-M. et al. Stretchable random lasers with tunable coherent loops. ACS Nano 9, 12436-12441 (2015)

47. Walpole, J. N., Kintzer, E. S., Chinn, S. R., Wang, C. A. \& Missaggia, L. J. High-power strained-layer InGaAs/AIGaAs tapered traveling wave amplifier. Appl. Phys. Lett. 61, 740-742 (1992).

48. Galfsky, T., Gu, J., Narimanov, E. E. \& Menon, V. M. Photonic hypercrystals for control of light-matter interactions. Proc. Natl Acad. Sci. 114, 5125-5129 (2017).

49. Yun-Feng, $X$. et al. High quality factor, small mode volume, ring-type plasmonic microresonator on a silver chip. J. Phys. B 43, 035402 (2010).

50. Slobozhanyuk, A. P. et al. Purcell effect in hyperbolic metamaterial resonators. Phys. Rev. B 92, 195127 (2015).

51. Ginzburg, P. et al. Spontaneous emission in non-local materials. Light Sci. Appl. 6 e16273 (2017).

52. Ford, G. W. \& Weber, W. H. Electromagnetic interactions of molecules with metal surfaces. Phys. Rep. 113, 195-287 (1984).

53. Lu, D. et al. Nanostructuring multilayer hyperbolic metamaterials for ultrafast and bright green ingan quantum wells. Adv. Mater. 30, 1706411 (2018).

54. Novotny, L. \& Hecht, B. Principles of Nano-optics Chap. 2 (Cambridge University Press, Cambridge, 2006).

55. Hu, H.-W. et al. Wrinkled 2D materials: a versatile platform for low-threshold stretchable random lasers. Adv. Mater. 29, 1703549 (2017).

56. Kataria, M. et al. Transparent, wearable, broadband, and highly sensitive upconversion nanoparticles and graphene-based hybrid photodetectors. ACS Photonics 5, 2336-2347 (2018).

57. Wang, F. et al. Tuning upconversion through energy migration in core-shell nanoparticles. Nat. Mater. 10, 968-973 (2011).

58. Lin, H.-I., Wuu, D.-S., Shen, K.-C. \& Horng, R.-H. Fabrication of an ultra-flexible ZnO nanogenerator for harvesting energy from respiration. ECS J. Solid State Sci. Technol. 2, 400-404 (2013).

59. Lin, H. I., Horng, R. H., Shen, K. C. \& Wuu, D. S. ZnO nanowires embedded in epoxy resin separating from the substrate for wearable electronics applications. IEEE Trans. Nanotechnol. 13, 458-463 (2014).

60. Li, Y. et al. Light-induced degradation of $\mathrm{CH}_{3} \mathrm{NH}_{3} \mathrm{Pbl}_{3}$ hybrid perovskite thin film. J. Phys. Chem. C 121, 3904-3910 (2017).

61. Conings, B. et al. Intrinsic thermal instability of methylammonium lead trihalide perovskite. Adv. Energy Mater. 5, 1500477 (2015).

62. Woo, $\mathrm{H}$. C. et al. Temperature-dependent photoluminescence of $\mathrm{CH}_{3} \mathrm{NH}_{3} \mathrm{PbBr}_{3}$ perovskite quantum dots and bulk counterparts. J. Phys. Chem. Lett. 9, 4066-4074 (2018).

63. Schmidt, L. C. et al. Nontemplate synthesis of $\mathrm{CH}_{3} \mathrm{NH}_{3} \mathrm{PbBr}_{3}$ perovskite nanoparticles. J. Am. Chem. Soc. 136, 850-853 (2014).

64. Johnson, P. B. \& Christy, R. W. Optical constants of the noble metals. Phys. Rev. B 6, 4370-4379 (1972)

65. Alias, M. S. et al. Optical constants of $\mathrm{CH}_{3} \mathrm{NH}_{3} \mathrm{PbBr}_{3}$ perovskite thin films measured by spectroscopic ellipsometry. Opt. Express 24, 16586-16594 (2016).

\section{ACKNOWLEDGEMENTS}

This work was financially supported by the "Advanced Research Center for Green Materials Science and Technology" from The Featured Area Research Center Program within the framework of the Higher Education Sprout Project by the Ministry of Education (108L9006) and the Ministry of Science and Technology in Taiwan (MOST 108-3017-F-002-002 and MOST 108-2112-M-002-019). H.I L. acknowledges fellowship support from the Ministry of Science and Technology Overseas Project for Post Graduate Research, Taiwan (108-2917-I-002-010).

\section{AUTHOR CONTRIBUTIONS}

H.I L. and C.C.W. contributed equally to this work. H.I L. and Y.F.C. conceived the idea of flexible and rollable HMM. H.I L. and C.C.W. prepared the HMM devices. H.I L., C.C. W., K.C.S., and Y.F.C. discussed the mechanism of HMM. M.Y.S. calculated the k-space 
dissipated power density. K.C.S. took the FE-SEM images. H.I L., M.K., and C.R.P.I. took the TEM image. P.K.R. and K.P.B. prepared the PNCS. H.I L. and C.C.W. measured emission spectra. H.I L. performed simulation. H.I L. and Y.F.C. discussed the mechanism of random laser action. Y.F.C. supervised the project. H.I L. and Y.F.C. wrote the paper. All the authors were involved in analyzing the data.

\section{COMPETING INTERESTS}

The authors declare no competing interests.

\section{ADDITIONAL INFORMATION}

Supplementary information is available for this paper at https://doi.org/10.1038/ s41528-020-00085-6.

Correspondence and requests for materials should be addressed to Y.-F.C.

Reprints and permission information is available at http://www.nature.com/ reprints
Publisher's note Springer Nature remains neutral with regard to jurisdictional claims in published maps and institutional affiliations.

Open Access This article is licensed under a Creative Commons By Attribution 4.0 International License, which permits use, sharing, adaptation, distribution and reproduction in any medium or format, as long as you give appropriate credit to the original author(s) and the source, provide a link to the Creative Commons license, and indicate if changes were made. The images or other third party material in this article are included in the article's Creative Commons license, unless indicated otherwise in a credit line to the material. If material is not included in the article's Creative Commons license and your intended use is not permitted by statutory regulation or exceeds the permitted use, you will need to obtain permission directly from the copyright holder. To view a copy of this license, visit http://creativecommons. org/licenses/by/4.0/.

(c) The Author(s) 2020 